Case Report

\title{
Intrapulmonary-located Castleman's Disease, Which Was Surgically Resected without Pulmonary Resection
}

\author{
Gulsah Gunluoglu, MD, ${ }^{1}$ Aysun Olcmen, MD, ${ }^{2}$ Sinem Nedime Sokucu, MD, ${ }^{1}$
}

Hasan Akin, MD, ${ }^{2}$ and Ibrahim Dincer, $\mathrm{MD}^{2}$

\begin{abstract}
Castleman's disease (CD) is a rare disease with unknown aetiology. It is characterised by benign lymph node hyperplasia that may involve all lymph nodes. The most common locations are the mediastinum and abdomen. CD arising from intrapulmonary lymph nodes has been reported in five cases, in the English language literature to date. Tumours in these patients are usually resected during lung surgery. An asymptomatic 29-year-old male patient was evaluated due to a mass lesion with a diameter of $55 \mathrm{~mm}$ located in the infrahilar region of the right lung with a high degree of contrast enhancement on thoracic computed tomography (CT). Vascularity of this central lesion was excluded by pulmonary angiography. Thoracotomy was performed due to the inability to obtain a diagnosis with percutaneous fine needle aspiration biopsies. A frozen section examination of the mass revealed a benign lesion, arising from the intrapulmonary lymph nodes and protruding to the lower-lobe parenchyma. The mass was then extracted from the parenchyma. After histopathological evaluation of the mass, CD involving the lymph nodes was diagnosed. CD rarely involves the intrapulmonary lymph nodes. Diagnosis is difficult in these patients, and thoracotomy may be required. After obtaining benign results by mass sampling, limited resection of these masses, while sparing the lung parenchyma, may be possible.
\end{abstract}

Keywords: Castleman's disease, intrapulmonary localization

\section{Introduction}

Castleman's disease (CD) is a term used for angiofollicular lymph node hyperplasia. It was first described in

${ }^{1}$ Departman of Chest Diseases, Yedikule Chest Diseases and Thoracic Surgery Education and Research Hospital, Istanbul, Turkey

${ }^{2}$ Departman of Thoracic Surgery, Yedikule Chest Diseases and Thoracic Surgery Education and Research Hospital, Istanbul, Turkey

Received: November 12, 2010; Accepted: December 17, 2010 Corresponding author: Gulsah Gunluoglu, MD. Bahcelievler mah, Aydinerler cad, Sakli Vadi konutlari, Erguvan blok No:17, Bahcelievler, Istanbul, Turkey

Email: gunluoglugulsah@yahoo.com.tr

(C)2011 The Editorial Committee of Annals of Thoracic and Cardiovascular Surgery. All rights reserved.
1954. ${ }^{1)}$ Two clinical patterns exist: localized (usually the hyaline vascular type) and multicentric (plasma cell type). The localized type is a rare form of lymphoid hyperplasia. It is usually incidentally detected in asymptomatic, young-to-middle-aged people as a mediastinal mass lesion. Along with mediastinal localization, pleural, chest wall, and extrathoracic involvement have also been reported. ${ }^{2)}$ On the other hand, intrapulmonary development of $\mathrm{CD}$ is very rare, and only five cases have been reported in the English language literature to date..$^{2-5)} \mathrm{In}$ all of these cases, mass lesions were resected by lobectomy. Here, we present an intrapulmonary-located CD case and its surgical treatment.

\section{Case}

A 29-year-old asymptomatic male patient was 


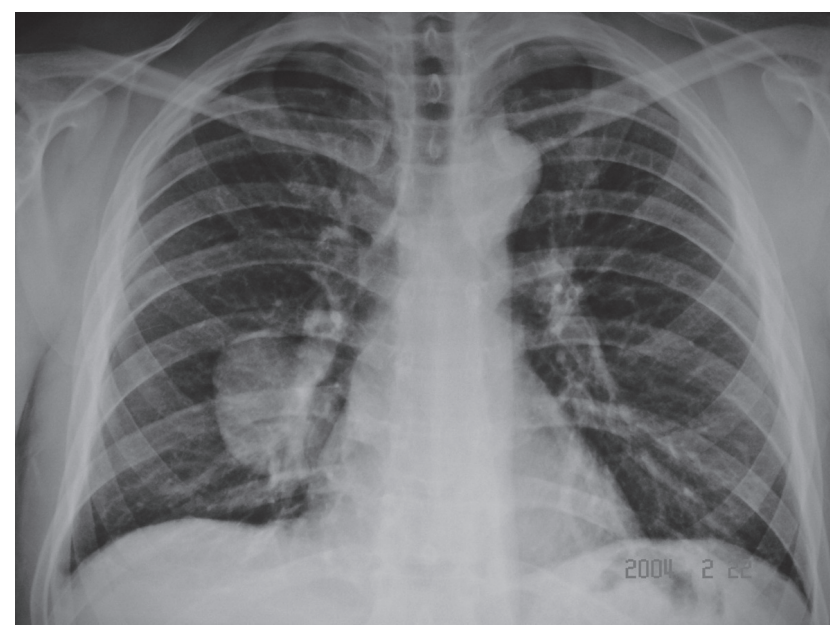

Fig. 1 P-A Chest X ray: Mass lesion located in the right hilerinfrahiler region.

admitted to our hospital with $5 \times 4-\mathrm{cm}$ tumour mass located in the right infrahilar region not obliterating the heart contours on his chest X-ray, which had been taken incidentally (Fig. 1). He was a non-smoker, and no pathological finding was detected on physical examination. His blood count and routine biochemical parameters were within normal limits. On thoracic CT, a $5.5 \times 4-\mathrm{cm}$ lesion was seen in his right lung with a hilar-infrahilar localization with high contrast enhancement (Fig. 2). Due to the high contrast enhancement and central localization, near the pulmonary artery, a pulmonary artery aneurysm could not be ruled out.

Conventional pulmonary angiography was performed because of the differential diagnosis, and no participation of the pulmonary vascular structures was seen. To determine more about the nature of the lesion and to assess the need for an invasive intervention, F-18 fluorodeoxyglucose (FDG)-labelled CT integrated positron emission tomography (PET/CT) was taken. The maximum standard FDG uptake level (SUD max) of the lesion in the lung was 2.9, and no other location with pathological FDG uptake was detected. Even with this result, lowgrade tumours, such as leiomyoma, carcinoid tumour, and mucoepidermoid carcinoma, as well as lung cancer, could still not be excluded from the differential diagnosis.

For diagnostic purposes, fiberoptic bronchoscopy was performed under local anesthesia. In the bronchoscopic examination, the lateral segment of the median lobe of the right bronchus system was occluded by a cleft-like compression. Transbronchial needle aspiration biopsies

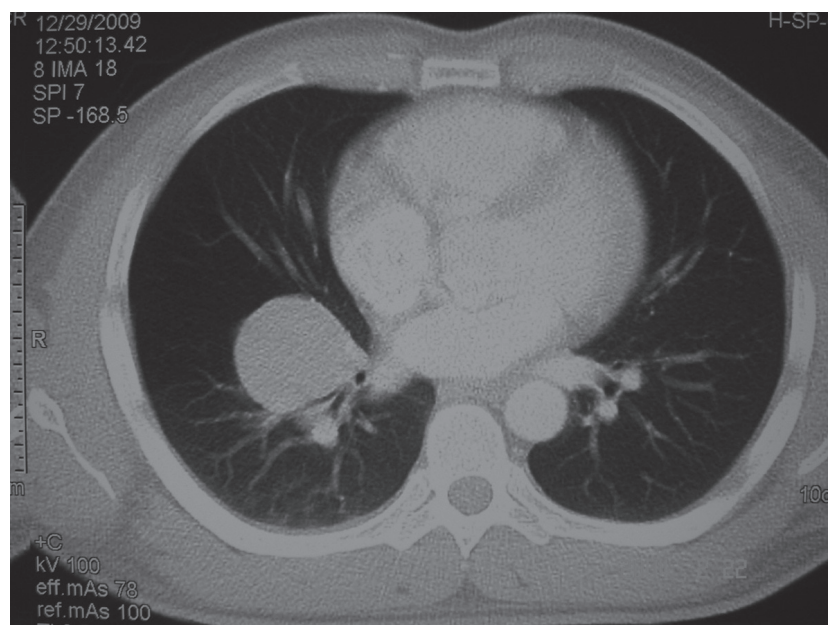

Fig. 2 Thorax tomography: $5.5 \times 4 \mathrm{~cm}$ mass lesion located in right hiler-infrahiler region with high contrast enhancement.

were performed at this location. No specific diagnosis was obtained from the cytological evaluation. Transthoracic fine needle aspiration biopsy of the lesion was then performed. Again, no specific diagnosis was obtained. After explanation of the preliminary diagnoses to the patient, he accepted thoracotomy as a further diagnostic approach.

In the thoracotomy exploration, a smoothly contoured lesion arising from the lymph nodes in the interlobar region and protruding to the lower lobe in the major fissure, which resulted in fibrosis in the interlobar region and adhered to the pulmonary artery in this location, was detected. Frozen section analysis of the incisional biopsy revealed a benign lymphatic development without malignant cells. Due to this result, limited resection was performed. Although the mass lesion showed dense and vascular adhesions to the lower lobe of the lung, these were dissected without significant bleeding. Fibrotic areas above the interlobar pulmonary artery were carefully separated from the vascular structures. Several bronchial artery branches in the interlobar area, which were supplying the mass, were detected during this dissection and cut using ligatures. The mass lesion was relieved from the surrounding structures and completely removed without any accompanying lung tissue or bronchial structures. The patient was discharged on the fifth postoperative day with no postoperative complication. Results of the histopathological evaluation revealed hyaline vascular type lymph node hyperplasia (Fig. 3). From this result, CD was diagnosed. 


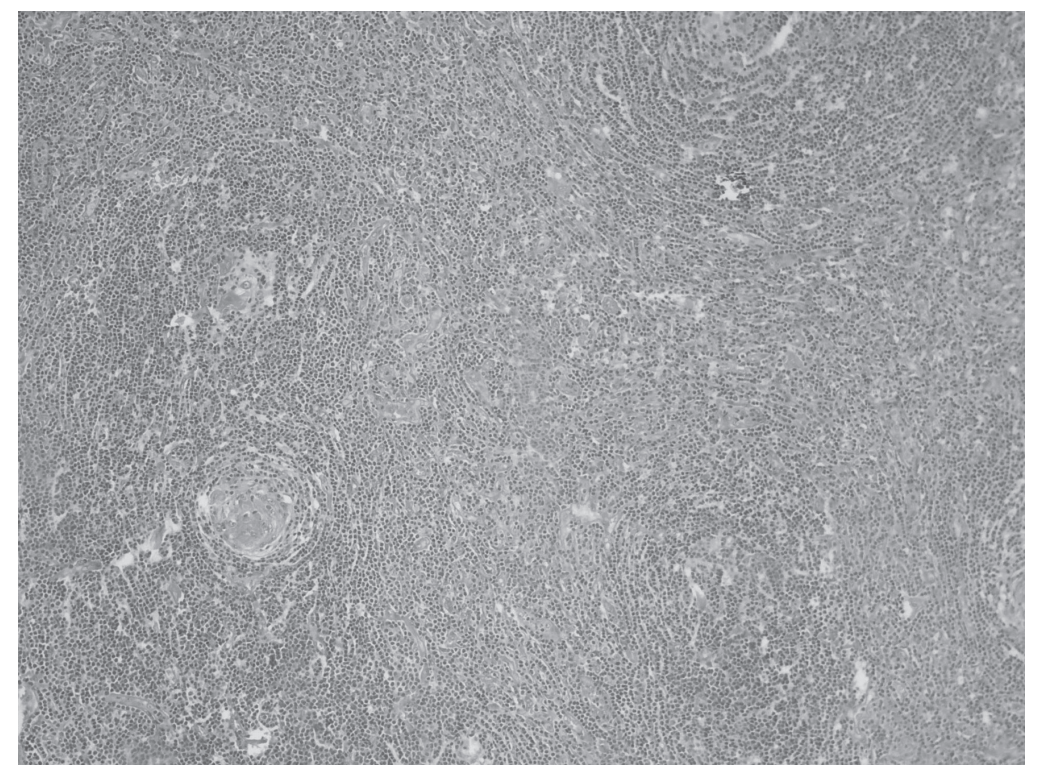

Fig. 3 (HEX200) Vascular structure with folliculer hyaline alteration and significant germinal center around them.

\section{Comments}

$\mathrm{CD}$ is a rarely seen lymphoproliferative disorder with unknown etiology. It may develop in any organ in the body. The most common sites are cervical (42\%), mediastinal (31\%), abdominal (18\%), and retroperitoneal (5\%). Extranodal involvement occurs in only $5 \%$ of patients. ${ }^{6}$ Intrapulmonary lymph node involvement in $\mathrm{CD}$ is very rare; only five cases have been reported in the English language literature. $\left.{ }^{2-5}\right)$ Keller et al. did not report the properties or treatment methods of their two cases. ${ }^{3)}$ When the other three cases were evaluated together with our case, the gender distribution was equal. The histopathologic type of each of these four cases was the hyaline vascular type. Our case was localized in the right lung, while the others were located in the left lung.

CD has two distinct clinical forms: the unicentric or localized form, and the multicentric or systemic form. Histopathologically, hyaline vascular, plasma cell, and mixed types exist. The localized form usually presents as the hyaline vascular type. It is primarily observed to be a slow-growing, asymptomatic mass lesion. ${ }^{6}$ In contrast to localized disease, the systemic form is characterized by generalized lymphadenopathy and systemic symptoms, such as fever, fatigue, and weight loss. ${ }^{7)}$

The most common radiological property of the hyaline vascular type is contrast enhancement on CT. ${ }^{8,9)}$ Pulmonary arterial aneurysm was included in the differential diagnosis in our case due to the vascularization and hilar localization of the lesion. The effectiveness of angiography in the differential diagnosis has been reported. ${ }^{8)}$ In light of this, we showed that our lesion had no vascular involvement. The value of PET/CT in the diagnosis is controversial because it was ineffective in differentiating between malignant disease and $\mathrm{CD}$; a low preoperative SUD max value detected on PET/CT could not exclude malignant disease in our case.

On the other hand, the lack of pathological involvement of the lymph nodes was useful in the surgical approach. Thoracotomy without mediastinal sampling was chosen for diagnosis and treatment. Also, we thought that PET/CT may be effective in the differentiation between localized and multifocal disease.

Treatment of localized CD requires surgery or radiotherapy. Corticosteroids and antineoplastic chemotherapy are indicated in systemic disease. Complete surgical excision in localized disease is reportedly curative in most cases. ${ }^{2)}$ Radiotherapy may be helpful in patients who are at risk of recurrence or who are not surgical candidates. ${ }^{10)}$ The intraoperative bleeding risk is reportedly high in hyaline vascular type tumours due to hypervascularity and adhesion of the tumour to the surrounding tissue. ${ }^{11)}$ In our case, the tumour adhered to the pulmonary arterial branches, as has been reported in the literature. When the case presentations in the literature were analyzed, the preferred surgical procedure was lobectomy. The reason 
for this could be the potential for bleeding of the tumour and the inability to differentiate malignancy at the time of resection. However, after seeing that the pathological examination of the frozen section of the tumour revealed benign cytology in our case, total excision of the tumour without major hemorrhage was possible.

\section{Conclusions}

Intrapulmonary $\mathrm{CD}$ should be kept in mind during the differential diagnosis of localized mass lesions, especially when the lesions are near the hilar region, in young, asymptomatic patients. The diagnosis is very difficult in these patients, and a thoracotomy is usually needed for the diagnosis and treatment. After obtaining benign results from sampling of the mass, limited resection of the mass while sparing the lung parenchyma may be possible.

\section{References}

1) Castleman B, Towne VW. Case records of the Massachusetts General Hospital case no.40011. N Engl J Med 1954; 250: 26-30.

2) Mohanna S, Sanchez J, Ferrufino JC, et al. Characteristics of Castleman's disease in Peru. Eur J Intern Med 2006; 17: 170-4.

3) Keller AR, Hochholzer L, Castleman B. Hyaline-vas- culer and plasma cell types of giant lymph node hyperplasia of the mediastinum and the other locations. Cancer 1972; 29: 670-83.

4) Yeh CM, Chou CM, Wong LC. Castleman's disease mimicking intrapulmonary malignancy. Ann Thorac Surg 2007; 84: e6-7.

5) Tokunaga T, Kadota Y, Utsumi T, et al. Castleman's disease arising from an intrapulmonary lymph node. Gen Thorac Cardiovasc Surg 2009; 57: 562-5.

6) Danon AD, Krishnan J, Frizzera G. Morpho-immunophenotypic diversity of Castleman's disease, hyalinevascular type: with emphasis on a stroma-rich variant and a new pathogenetic hypothesis. Virchows Arch A Pathol Anat Histopathol 1993; 423: 369-82.

7) Frizzera G, Peterson BA, Bayrd ED, et al. A systemic lymphoproliferative disorder with morphologic features of Castleman's disease: clinical findings and clinicopathologic correlations in 15 patients. J Clin Oncol 1985; 3: 1202-16.

8) McAdams HP, Rosado-de-Christenson M, Fishback $\mathrm{NF}$, et al. Castleman disease of the thorax: radiologic features with clinical and histopathologic correlation. Radiology 1998; 209: 221-8.

9) Khan J, von Sinner W, Akhtar M, et al. Castleman's disease of the chest. Magnetic resonance imaging features. Chest 1994; 105: 1608-10.

10) Gaba AR, Stein RS, Sweet DL, et al. Multicentric giant lymph node hyperplasia. Am J Clin Pathol 1978; 69: $86-90$.

11) Kurai M, Kondo R, Kobayashi N, et al Castleman's disease arising from the chest wall. Jpn J Thorac Cardiovasc Surg 2006; 54: 555-7. 